aim of treating hypotension is to preserve adequate end-organ perfusion and to avoid low cerebral blood flow which in premature infants can be associated with Intra-Ventricular Haemorrhage, Periventricular Leukomalacia and ultimately adverse neuro-developmental outcomes. The objective of this study, was to determine the pattern of inotrope and vasopressor use at a tertiary care Neonatal Intensive Care Unit (NICU) over a ten-year period from 2008 to 2017.

Methods We conducted a retrospective cross-sectional study over a ten-year period. The data gathered included the number of vials of dopamine, dobutamine, adrenaline, noradrenaline and hydrocortisone registered by the Pharmacy department to the NICU. From these figures, we extrapolated the pattern of pressor usage. We also reviewed the pattern of surfactant and antenatal steroid administration.

Results This study highlights that dopamine remains the firstline agent, regardless of the clinical scenario. Dobutamine is less commonly used, but figures remain high. An increase in endogenous catecholamine usage in 2013-2015 is apparent, with the administration of hydrocortisone tapering off in recent years. Rates of surfactant administration have decreased since 2013, coinciding with an ongoing upward trend in antenatal steroid administration to women up to 33 completed weeks of gestation

Discussion Low systemic blood flow is commonly encountered in extremely premature infants. Despite this, it remains unclear what the safest and most effective drug is to prevent and manage hypotension. Few controlled trials have directly compared the individual agents and the effects that these drugs have on any meaningful outcome. As such, the database with the highest level of medical evidence, the Cochrane Library has come to few solid clinical recommendations.

Conclusion Despite the ongoing upward trend in admissions to NICU, inotrope and vasopressor use is falling. We hypothesise that this reflects an overall improvement in the management of newborns, as pressor agents act as a surrogate marker of a baby's underlying condition. Through the judicious use of antenatal steroids, greater attention to initial resuscitation, early initiation of CPAP and early extubation, survival rates are improving, incidence of Cystic PVL is falling and overall morbidity is down

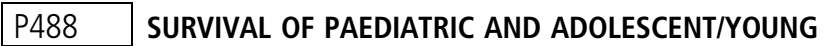 ADULT (AYA) CANCER PATIENTS WITH SARCOMA IN IRELAND DURING 1994-2014: COMPARISONS BY AGE}

\begin{abstract}
${ }^{1}$ Scheryll Alken*, ${ }^{2}$ Charles Gilham, ${ }^{1}$ Michael Capra, ${ }^{3}$ Cliona Grant, 'Jane Pears, ${ }^{4}$ Sandra Deady, ${ }^{5}$ Deirdre O'Mahony, ${ }^{4}$ Paul Walsh, ${ }^{1}$ Cormac Owens. ${ }^{1}$ Our Lady's Children's Hospital, Crumlin, Dublin, Ireland; '2St Luke's Radiation Oncology Network, Dublin, Ireland; ${ }^{3}$ St James's Hospital, Dublin, Ireland; ${ }^{4}$ National Cancer Registry Ireland, Cork, Ireland; ${ }^{5}$ Cork University Hospital, Cork, Ireland
\end{abstract}

\subsection{6/archdischild-2019-epa.824}

Some studies indicate that survival of AYAs with cancer may be inferior to that of younger children with similar cancers, possibly related (in part) to differences in access to centralised or standardised treatments. We sought to examine the comparative survival of paediatric \& AYA patients in Ireland across a 20-year period.

Methods Using the National Cancer Registry Ireland (NCRI) database, all patients diagnosed with a sarcoma age 0-24 between 1994-2014 were identified. Survival was based on matching of cases against national death certificate data complete up to 31 December 2014 \&amp grouped in 2 diagnostic cohorts: 1994-2003 and 2004-2014 and examined according to the ICCC.

Results 577 patients less than 25 years were diagnosed with a sarcoma between 1994-2014; 321 under 15. Significantly poorer survival was noted for the AYA patients compared with paediatric patients for the following sarcoma groups and diagnosis periods

- Malignant bone tumours, 1994-2013 (EHR 1.62, 1.05-2.48, $\mathrm{P}=0.026$ ) and 1994-2003 (EHR 1.87, 1.05-3.33, $\mathrm{P}=0.032$ );

- Ewing \& related tumours), 1994-2013 (EHR 2.04, 95\% CI 1.11-3.72, $\mathrm{P}=0.021)$ and 1994-2003 (EHR 2.68, 95\% CI 1.22-5.87, $\mathrm{P}=0.014)$;

- Soft tissue sarcomas), 1994-2013 (EHR 2.14, 1.29-3.53, $\mathrm{P}=0.03$ ) and 2004-2013 (EHR 2.52, 1.20-5.25, $\mathrm{P}=0.014$ ).

Conclusions This study highlights the disparities that exist in outcomes for AYA patients with sarcomas treated in Ireland. The exact cause for this is unclear and is likely multifactorial, possibly owing to lack of standardised/centralised services. Future development on a national level is imperative.

\section{P489 CANCER PREDISPOSITION SCREENING IN CHILDREN WITH CANCER IN IRELAND}

${ }^{1}$ Siobhan Burrington*, 'Lesley Darcy, ${ }^{2}$ Terri McVeigh, 'Michael Capra. 'Our Lady's Children's Hospital Crumlin, Dublin, Ireland; ${ }^{2}$ Royal Marsden NHS Foundation Trust, London, UK

\subsection{6/archdischild-2019-epa.825}

Background Presently, 8-10\% of children and adolescents diagnosed with cancer have an underlying cancer predisposition syndrome, however the true figure may be higher. Family history alone identifies $<4 \%$ of such patients and is therefore not sufficient in isolation as de novo mutations also occur. It is important to identify patients with cancer predisposition syndromes to guide further screening and tailor long term follow up and surveillance programmes. In addition, such information may help counsel family members on cancer risk. At present we believe we are under-referring patients for genetic cancer predisposition testing. The Royal Marsden Hospital is piloting a new system of identifying childhood and adolescent cancer patients for genetic screening.

Aim To document how many patients at Our Lady's Children's Hospital, Crumlin, relevant to their specific cancer diagnosis, may benefit from genetic referral to identify those who may have an underlying cancer predisposition syndrome

Methods Retrospective review of all patients diagnosed with cancer in OLCHC between 01/01/2017 - 31/12/17.

Using the Royal Marsden Hospital 'Stop-light' system relative to the patient's diagnosis, patients who have a diagnosis labelled as:

1. Red - automatically eligible for genetic referral

2. Yellow - may benefit from referral

3. Green - do not require referral

Results There were 160 patients diagnosed in the period 01/ $01 / 2017$ - 31/12/2017. Of these, 6 were excluded because of a pre-cancerous, rather than cancer, diagnosis: aplastic 